\title{
Uterine Fibroid Embolisation for Symptomatic Uterine Fibroids: A Survey of Clinical Practice in Europe
}

\author{
Marianne J. Voogt $\cdot$ Mark J. Arntz • \\ Paul N. M. Lohle $\cdot$ Willem P. Th. M. Mali • \\ Leo E. H. Lampmann
}

Received: 5 May 2010/Accepted: 20 August 2010/Published online: 21 September 2010

(C) The Author(s) 2010. This article is published with open access at Springerlink.com

\begin{abstract}
Purpose To assess current uterine fibroid embolisation (UFE) practice in European countries and determine the clinical environment for UFE in different hospitals.

Material and Methods In May 2009, an invitation for an online survey was sent by e-mail to all members of the Cardiovascular and Interventional Radiologic Society of Europe, representing a total number of 1,250 different candidate European treatment centres. The survey covered 21 questions concerning local UFE practice.

Results A total of 282 respondents completed the questionnaire. Fifteen questionnaires were excluded because they were doubles from centres that had already returned a questionnaire. The response rate was 267 of 1,250 centres (21.4\%). Ninety-four respondents (33\%) did not perform UFE and were excluded, and six centres were excluded because demographic data were missing. The remaining 167 respondents from different UFE centres were included in the study. Twenty-six percent of the respondents were from the United Kingdom $(n=43) ; 16 \%$ were from Germany $(n=27) ; 11 \%$ were from France $(n=18)$; and the remaining $47 \%(n=79)$ were from other European countries. Most centres $(48 \%, n=80)$ had 5 to 10 years
\end{abstract}

M. J. Voogt ( $₫)$. W. P. Th. M. Mali

Department of Radiology, University Medical Centre Utrecht, P.O. Box 85500, 3508 GA Utrecht, The Netherlands

e-mail: m.voogt@umcutrecht.nl

M. J. Arntz

Department of Radiology, University Medical Centre Nijmegen, P.O. Box 9101, 6500 HB Nijmegen, The Netherlands

P. N. M. Lohle · L. E. H. Lampmann

Department of Radiology, Sint Elisabeth Hospital,

P.O. Box 90151, 5000 LC Tilburg, The Netherlands experience with UFE and performed 10 to 50 procedures annually (53\% $[n=88])$ of respondents). Additional demographic data, as well as specific data on referral of patients, UFE techniques used, and periprocedural and postprocedural, care will be provided.

Conclusion Although UFE as an alternative treatment for hysterectomy or myomectomy is widespread in Europe, its impact on the management of the patient with symptomatic fibroids seems, according to the overall numbers of UFE procedures, somewhat disappointing. Multiple factors might be responsible for this observation.

Keywords Uterine fibroids · Uterine fibroid embolisation · Survey

\section{Introduction}

Uterine fibroids are the most common benign tumours in women of childbearing age. Symptomatic fibroids can cause a diversity of symptoms, which can be divided into four categories: bleeding symptoms (irregular and/or heavy menstrual bleeding), pain (in the pelvic region and the back), bulk-related symptoms (pressure on bladder and bowel as well as increase in abdominal circumference), and subfertility [1]. These symptoms often lead to medical or surgical treatments.

During the last two decades, minimally invasive therapeutic options for uterine fibroids have increased considerably. Uterine fibroid embolisation (UFE) was introduced in 1994 and is currently a well acknowledged and proven alternative to surgical treatment $[2,3]$.

UFE is a percutaneous trancatheter embolisation technique using embolisation material to occlude the (end-)arteries supplying the fibroid. Devascularisation 
causes infarction and consequently decreased fibroid size, which may result in effective alleviation of symptoms.

However, after the introduction of UFE as an alternative to more invasive approaches, a real widespread breakthrough, especially in general interventional radiology (IR), did not occur.

Information on UFE still seems to be unavailable to a large number of women in Europe, and many gynaecologists do not provide the option of UFE, or they inaccurately inform patients, using misleading facts. Specific data on the number of centres and interventionalists performing UFE in Europe and the number of UFE procedures per centre do not exist.

This publication reports the outcome of a survey among European interventional radiologists concerning UFE treatment. The purpose of this study was to determine actual data on the current clinical practice of UFE in European countries.

\section{Materials and Methods}

In May 2009, we designed a survey to assess current UFE practice in European countries. All professionally active European members of the Cardiovascular and Interventional Radiologic Society of Europe (CIRSE) were invited by e-mail to participate in this study. The total number of different candidate treatment facilities in Europe was 1,250. The questionnaire consisted of 21 questions concerning local clinical practice of UFE and related topics (see Appendix for complete questionnaire). The online button-driven questionnaire was designed for easy handling with a simple set-up to be sure that as many interventional radiologists as possible would participate in this study and could be able to complete all fields.

The first questions referred to demographic data concerning the treatment facility in which respondents were working. The next question was if UFE was performed by the responding interventional radiologist. In case the answer was "no," the questionnaire was excluded from the database. The remaining respondents were asked about the interventional group in their facility (number of interventional radiologists and how many of them performed UFE). Respondents had to indicate the time period when their hospital staff started performing UFE as well as the number of UFE procedures performed per year. Respondents were asked to describe the referral pattern of patients (self-referral, e.g., directly to IR, referral by gynaecologist, or referral by general practitioner) categorized as percentages adding up to $100 \%$. The questionnaire also assessed who was responsible for preprocedural and postprocedural patient care (radiologist, gynaecologist, or both). The type of pain management, such as patient-controlled analgesia
(PCA), epidural analgesia, or other-as well as the duration of hospitalisation-were also inventoried. Specific procedure-related questions, such as preferred vascular access (unifemoral, bifemoral, or other), use of microcatheters, type of embolic agents (gelatin sponge, spherical or nonspherical embolic material) used, and the advocated embolisation end point (complete stasis, sluggish flow, or pruned tree appearance), were asked. The use of magnetic resonance imaging (MRI) for preprocedural and postprocedural evaluation was also discussed. The last items were aimed at the future expectations of the respondents concerning Magnetic Resonance-guided Focused Ultrasound (MRgFUS) as a new treatment alternative for uterine fibroids, and we asked if the treatment facility had a Web site to allow screening of the Web sites for dedicated information on UFE treatment. Participants were asked to complete the online questionnaire before the end of July 2009. To avoid bias, we decided to include only one survey per treatment centre. In case more than one questionnaire was returned from the same facility, we decided to include the first submitted survey in the study and exclude the duplicates.

\section{Results}

A total of 282 respondents returned the completed questionnaire (Table 1). Fifteen questionnaires were excluded because they were duplicates from treatment centres that had already returned a questionnaire. The response rate was therefore 267 of 1,250 candidate treatment centres (21.4\%). Ninety-four respondents (33\%) did not perform UFE and were consequently excluded from the study. Six questionnaires were excluded because essential demographic data were missing. Further contact efforts to obtain these missing data did not result in sufficient completion of the information, thus making rejection of these questionnaires inevitable. The remaining 167 respondents, all from different UFE centres, were included in the study. The geographic distribution of the respondents covered 24 countries in Europe, including Turkey. Figure 1 shows the number of included treatment facilities per country.

Twenty-six percent $(n=43)$ of the respondents were from the United Kingdom; $16 \%(n=27)$ were from Germany; $11 \%(n=18)$ were from France; and the remainder $(53 \%, n=79)$ were from other European countries. Fifty-two percent $(n=86)$ of the respondents worked in an academic centre, and the remaining $48 \%$ $(n=81)$ worked in a general hospital setting or private practice. The majority of the respondents $(65 \%, n=108)$ worked in a group with $\geq 1$ interventional radiologist performing UFE. Only $1 \%(n=2)$ of the respondents had $>15$ years of experience with UFE; $25 \%(n=42)$ had $10-15$ years of experience; the majority $(48 \%, n=80)$ had 

centres per European country and response rate
Table 1 Number of treatment

\begin{tabular}{|c|c|c|c|c|c|}
\hline \multirow[t]{2}{*}{ Countries } & \multirow{2}{*}{$\begin{array}{l}\text { No. of treatment } \\
\text { centres }\end{array}$} & \multirow{2}{*}{$\begin{array}{l}\text { No. of } \\
\text { respondents }\end{array}$} & \multicolumn{2}{|c|}{ No. of exclusions } & \multirow{2}{*}{$\begin{array}{l}\text { No. of included } \\
\text { centres }(\%)\end{array}$} \\
\hline & & & No UFE & Duplicates & \\
\hline Austria & 54 & 14 & 5 & 0 & $9(16.7)$ \\
\hline Belgium & 27 & 8 & 2 & 1 & $5(18.5)$ \\
\hline Bulgaria & 7 & 2 & 1 & 0 & $1(14.3)$ \\
\hline Croatia & 6 & 0 & 0 & 0 & $0(0)$ \\
\hline Cyprus & 1 & 0 & 0 & 0 & $0(0)$ \\
\hline Czech Republic & 35 & 2 & 2 & 0 & $0(0)$ \\
\hline Denmark & 9 & 5 & 3 & 0 & $2(22.2)$ \\
\hline Finland & 15 & 3 & 1 & 0 & $2(13.3)$ \\
\hline France & 85 & 20 & 0 & 2 & $18(21.2)$ \\
\hline Germany & 289 & 37 & 9 & 1 & $27(9.3)$ \\
\hline Greece & 67 & 18 & 15 & 0 & $3(4.5)$ \\
\hline Hungary & 19 & 2 & 0 & 0 & $2(10.5)$ \\
\hline Iceland & 1 & 0 & 0 & 0 & $0(0)$ \\
\hline Ireland & 14 & 9 & 2 & 1 & $6(42.9)$ \\
\hline Italy & 50 & 12 & 6 & 0 & $6(12.0)$ \\
\hline Latvia & 1 & 0 & 0 & 0 & $0(0)$ \\
\hline Luxembourg & 6 & 1 & 1 & 0 & $0(0)$ \\
\hline Malta & 1 & 0 & 0 & 0 & $0(0)$ \\
\hline Norway & 23 & 9 & 3 & 0 & $6(26.1)$ \\
\hline Poland & 27 & 3 & 0 & 2 & $1(3.7)$ \\
\hline Portugal & 11 & 2 & 1 & 0 & $1(9.1)$ \\
\hline Romania & 6 & 5 & 0 & 4 & $1(16.7)$ \\
\hline Russia & 14 & 1 & 0 & 0 & $1(7.1)$ \\
\hline Serbia & 7 & 1 & 1 & 0 & $0(0)$ \\
\hline Slovakia & 4 & 1 & 0 & 0 & $1(25.0)$ \\
\hline Slovenia & 6 & 1 & 0 & 0 & $1(16.7)$ \\
\hline Spain & 52 & 12 & 1 & 1 & $10(19.2)$ \\
\hline Sweden & 13 & 4 & 1 & 0 & $3(23.1)$ \\
\hline Switzerland & 42 & 11 & 6 & 1 & $4(9.5)$ \\
\hline The Netherlands & 109 & 30 & 16 & 1 & 13 (11.9) \\
\hline Turkey & 61 & 6 & 5 & 0 & $1(1.6)$ \\
\hline Ukraine & 1 & 0 & 0 & 0 & $0(0)$ \\
\hline United Kingdom & 189 & 57 & 13 & 1 & $43(22.8)$ \\
\hline Missing demographic data & & 6 & & & \\
\hline Total & 1,250 & 282 & 94 & 15 & $167(13.4)$ \\
\hline
\end{tabular}

5-10 years of experience; and 26\% $(n=43)$ had introduced UFE during the last 5 years.

Table 2 lists the number of UFE procedures, per year and per country, classified into five categories: $\leq 0,10$ to 50,50 to 100,100 to 200 , and $\geq 200$ UFE treatments annually. Most centres $(53 \%, n=88)$ performed between 10 and 50 treatments on an annual basis. Extreme numbers of UFE treatments were provided by two treatment facilities (one in France and one in Romania): They both performed approximately 500 procedures/year.

In Table 3, data on preprocedural, periprocedural, and postprocedural care management are listed. In the majority of cases $(76 \%)$, patients were referred by a gynaecologist for UFE, and only in a small minority (4\%) were referred by a general practitioner. In the remaining cases $(20 \%)$, patients referred themselves directly to the interventional radiologist. Preprocedural care was generally performed by a combination of a gynaecologist and radiologist (42\%, $n=70$ ). Preprocedural MRI was considered a standard procedure by $56 \%(n=90)$ of the responding radiologists. Pain management was preferably performed $(76 \%$, $n=122)$ using PCA. Some facilities $(15 \%, n=24)$ used epidural analgesia, and 9\% $(n=14)$ employed other painmanagement protocols, such as intravenous medication $(n=8)$, oral medication $(n=1)$, a combination of PCA and epidural analgesia $(n=2)$, general anaesthesia 
Fig. 1 Number of included treatment facilities per European country

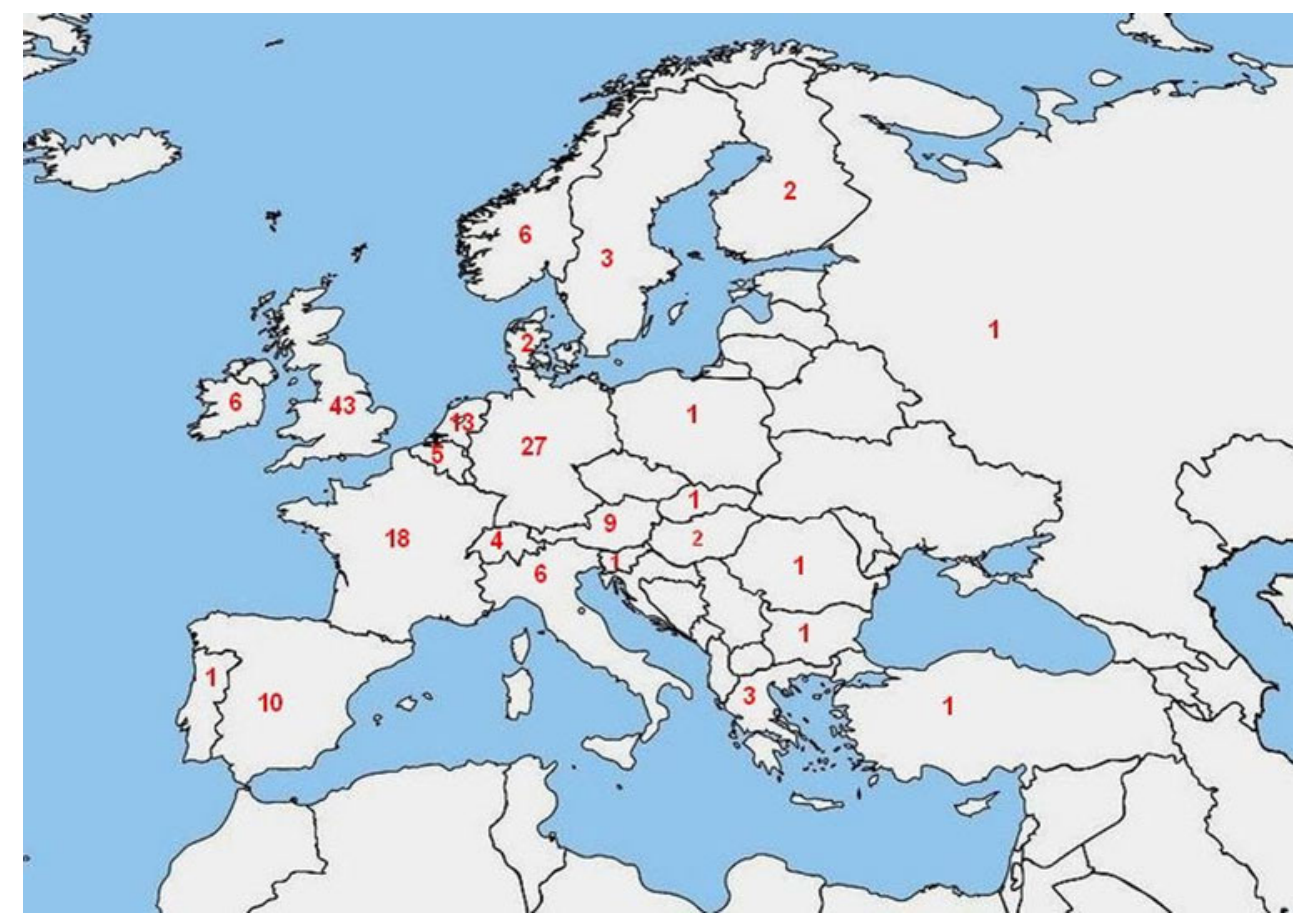

( $n=1)$, superior hypogastric plexus nerve block $(n=1)$, and neuroleptic medication $(n=1)$. Half of the respondents $(50 \%, n=81)$ admitted their patients for one overnight stay after UFE treatment. Only $1 \% \quad(n=1)$ performed UFE on an outpatient basis, and 3\% $(n=5)$ admitted their patients for a total of four overnight stays. Postprocedural care was most frequently $(55 \%, n=91)$ provided by a combination of a gynaecologist and radiologist. The majority of respondents $(72 \%, n=120)$ followed-up their patients up for 3 or 6 months, and $8 \%$ $(n=14)$ did so for $>12$ months.

In Table 4, UFE procedure-related details are listed. A large majority $(81 \%, n=134)$ of the respondents preferred unifemoral arterial access, whereas $17 \%(n=28)$ chose bifemoral arterial puncture, and only $2 \%(n=3)$ preferred brachial arterial access. Spherical embolic material was the favoured embolic agent in $77 \%$ ( $n=127)$ of the responding radiologists. The frequency of using microcatheters during UFE procedures varied among the respondents. Only 3\% $(n=5)$ indicated that they never use them; $36 \%(n=60)$ use them only when considered necessary; and $34 \%(n=56)$ always employ microcatheters during UFE. Fourteen percent ( $n=24)$ used the so-called "pruned tree" appearance on fluoroscopic imaging as the UFE end point. The user frequency of the end points "complete stasis" and "sluggish flow" was quite similar ( $41 \%, n=68$ vs. $45 \%, n=74)$.

Ninety percent $(n=151)$ of the treatment facilities had a Web site. We screened these Web pages for patient information on UFE. Nineteen percent $(n=31)$ contained dedicated passive or interactive treatment information for patients as well as physicians. Table 5 lists the annual number of treatments per centre in five categories for centres with and without a dedicated UFE Web site. This table illustrates that centres providing UFE information on a Web site have higher treatment numbers than facilities not operating an active Web site.

Future expectations for MR-HIFU as a thermoablative therapy for fibroids were indicated as "very promising" by five $\%(n=9)$ of the respondents; $30 \%(n=50)$ thought it was "promising," and 28\% $(n=47)$ did not see an important role for this treatment in the future. The remaining 36\% ( $n=60$ ) had no opinion about this topic.

\section{Discussion}

Since the publications of Ravina et al. in the early 1990s on premyomectomy transcatheter embolisation to minimize blood loss during surgery, the surprising effects on decreased fibroid size and symptoms became evident and led to the first reports on UFE as a single treatment for symptomatic fibroids [2, 4]. After a worldwide introduction as a possible alternative to hysterectomy and many additional studies on this treatment, UFE now seems to fit into the treatment options available to women suffering from uterine fibroids.

The Cochrane review from 2006 cited that UFE resulted in the same patient satisfaction rate as surgery (myomectomy or hysterectomy) [5]. The length of hospital stay was decreased after UFE, and the return to daily activities was faster. Because UFE seemed to result in a higher minor complication rate and more unscheduled visits and readmission rates, the statement was made that additional focus 
Table 2 Annual number of UFE procedures (classified into five categories) performed in treatment facilities per country

$n$ Number of treatment facilities

\begin{tabular}{|c|c|c|c|c|c|}
\hline Countries $(n)$ & $\leq 10$ & 10 to $<50$ & 50 to $<100$ & 100 to $<200$ & $\geq 200$ \\
\hline Austria $(n=9)$ & 2 & 7 & 0 & 0 & 0 \\
\hline Belgium $(n=5)$ & 1 & 4 & 0 & 0 & 0 \\
\hline Bulgaria $(n=1)$ & 1 & 0 & 0 & 0 & 0 \\
\hline Denmark $(n=2)$ & 0 & 2 & 0 & 0 & 0 \\
\hline Finland $(n=2)$ & 1 & 1 & 0 & 0 & 0 \\
\hline France $(n=18)$ & 5 & 10 & 1 & 1 & 1 \\
\hline Germany $(n=27)$ & 11 & 14 & 1 & 1 & 0 \\
\hline Greece $(n=3)$ & 2 & 1 & 0 & 0 & 0 \\
\hline Hungary $(n=2)$ & 0 & 0 & 2 & 0 & 0 \\
\hline Ireland $(n=6)$ & 1 & 3 & 1 & 1 & 0 \\
\hline Italy $(n=6)$ & 6 & 0 & 0 & 0 & 0 \\
\hline Norway $(n=6)$ & 3 & 3 & 0 & 0 & 0 \\
\hline Poland $(n=1)$ & 0 & 0 & 1 & 0 & 0 \\
\hline Portugal $(n=1)$ & 0 & 1 & 0 & 0 & 0 \\
\hline Romania $(n=1)$ & 0 & 0 & 0 & 0 & 1 \\
\hline Russia $(n=1)$ & 1 & 0 & 0 & 0 & 0 \\
\hline Slovakia $(n=1)$ & 0 & 1 & 0 & 0 & 0 \\
\hline Slovenia $(n=1)$ & 1 & 0 & 0 & 0 & 0 \\
\hline Spain $(n=10)$ & 4 & 6 & 0 & 0 & 0 \\
\hline Sweden $(n=3)$ & 2 & 1 & 0 & 0 & 0 \\
\hline Switzerland $(n=4)$ & 3 & 1 & 0 & 0 & 0 \\
\hline The Netherlands $(n=13)$ & 7 & 5 & 0 & 1 & 0 \\
\hline Turkey $(n=1)$ & 1 & 0 & 0 & 0 & 0 \\
\hline United Kingdom $(n=43)$ & 8 & 28 & 5 & 1 & 1 \\
\hline $\begin{array}{l}\text { Total number }(\%) \text { of treatment } \\
\text { facilities }(n=167)\end{array}$ & $60(36)$ & $88(53)$ & $11(6)$ & $5(3)$ & $3(2)$ \\
\hline
\end{tabular}

on long-term follow-up was necessary to determine the real impact of UFE [6].

As a result, retrospective cohort studies examined and compared the results of UFE and hysterectomy, which lead to satisfying conclusions concerning safety, expectations, and cost-effectiveness over a longer follow-up period [7, 8]. Furthermore, randomized studies comparing UFE and surgery (hysterectomy or myomectomy) have been completed in the meantime, resulting in publications in major scientific journals [9-12]. However, in gynaecological papers, the experimental character of UFE was only recently abandoned. Short, medium, and long-term followup data are currently available, leading to a more positive attitude from our gynaecological colleagues [3, 13, 14].

Guidelines from different medical specialities currently consent that UFE is a valuable alternative to surgical management of symptomatic fibroids in carefully selected and informed patients $[6,15]$. Bratby et al. quoted that level 1 evidence has established the role of UFE as a proper alternative treatment [16]. Last but not least, Bradley et al. stated that UFE is effective, safe, and durable and should be considered a true alternative to hysterectomy [3]. Apparently, the Cochrane review from 2006 is considerably outdated and must be revised as soon as possible.
This current European survey showed interesting findings on clinical UFE practice in a variety of treatment centres in different European countries. It was nevertheless interesting to discover the marked variation in current UFE practice across European centres in terms of distribution, approach, treatment care, and numbers. The top five of countries with the highest number of UFE centres were (starting with the highest) the United Kingdom, Germany, France, The Netherlands, and Spain. UFE facilities were not exclusively restricted to academic centres (52\%), as probably could be expected, but were also present in general hospital and/or private settings. Our survey also illustrates that although in 2009 UFE was widespread throughout European countries, the majority of centres (53\%) performed only between 10 and 50 UFE procedures/year. Only $5 \%$ performed $>100$ cases annually. Most respondents $(65 \%)$ were active in a group of interventionalists performing UFE, providing potential 24-h/7-day coverage of patient care. The overall impression that UFE is not new in Europe was expressed by the fact that the majority of centres (74\%) had $>5$ years of experience with UFE.

Participation with gynaecologists was performed by the majority of interventionalists in both preprocedural $(42 \%)$ and postprocedural $(55 \%)$ patient care. Preprocedural and 
Table 3 Preprocedural, periprocedural, and postprocedural carerelated information

\begin{tabular}{|c|c|c|}
\hline & $\%$ & $N$ \\
\hline \multicolumn{3}{|l|}{ Referral for UFE $(n=165)$} \\
\hline Self-referral & 20 & - \\
\hline Gynaecologist & 76 & - \\
\hline General practitioner & 4 & - \\
\hline \multicolumn{3}{|l|}{ Preprocedural care $(n=166)$} \\
\hline Radiologist & 17 & 28 \\
\hline Gynaecologist & 41 & 68 \\
\hline Combination & 42 & 70 \\
\hline \multicolumn{3}{|l|}{ Pain management $(n=160)$} \\
\hline Patient-controlled analgesia (PCA) & 76 & 122 \\
\hline Epidural analgesia & 15 & 24 \\
\hline Other & 9 & 14 \\
\hline \multicolumn{3}{|l|}{ Length of hospital stay $(n=163)$} \\
\hline Outpatient treatment & 1 & 1 \\
\hline 1 night & 50 & 81 \\
\hline 2 nights & 33 & 54 \\
\hline 3 nights & 13 & 22 \\
\hline 4 nights & 3 & 5 \\
\hline \multicolumn{3}{|l|}{ Postprocedural care $(n=166)$} \\
\hline Radiologist & 21 & 35 \\
\hline Gynaecologist & 24 & 40 \\
\hline Combination & 55 & 91 \\
\hline \multicolumn{3}{|c|}{ Postprocedural follow-up schedule $(n=166)$} \\
\hline No follow-up & 4 & 6 \\
\hline Follow-up for 3 months & 41 & 69 \\
\hline Follow-up for 6 months & 31 & 51 \\
\hline Follow-up for 12 months & 16 & 26 \\
\hline Follow-up for $>12$ months & 8 & 14 \\
\hline \multicolumn{3}{|l|}{ Planned MRI scan $(n=161)$} \\
\hline Preprocedure & 56 & 90 \\
\hline 3 months postprocedure & 18 & 29 \\
\hline 6 months postprocedure & 21 & 34 \\
\hline 9 months postprocedure & 0 & 0 \\
\hline 12 months postprocedure & 4 & 7 \\
\hline$>12$ months postprocedure & 1 & 1 \\
\hline
\end{tabular}

$n$ Number of respondents who answered the specific question

postprocedural care solely by IR was noted in only 17 and $21 \%$ of centres, respectively. Gynaecologists were the main referrers for UFE treatment. The preferred painmanagement in the majority of centres (76\%) was PCA, as expected, and most centres (50\%) admitted the patients for one overnight stay after the procedure.

In terms of facility-related efficacy estimation, it was disappointing to note that only $24 \%$ of centres followedup their patients for $\geq 12$ months, $31 \%$ for only 6 months, and the majority (41\%) for only 3 months. Although he use of preprocedural and postprocedural MRI is
Table 4 UFE procedure-related information

\begin{tabular}{lrr}
\hline & $\%$ & $N$ \\
\hline Preferred arterial access $(n=165)$ & 81 & 134 \\
Unifemoral & 17 & 28 \\
Bifemoral & 2 & 3 \\
Brachial & & \\
Embolic agent $(n=166)$ & 1 & 2 \\
Gelatin sponge & 77 & 127 \\
Spherical embolic material & 22 & 37 \\
Nonspherical embolic material & & 5 \\
Use of microcatheters $(n=166)$ & 3 & 60 \\
Never & 36 & 45 \\
Seldom & 27 & 56 \\
Regularly & 34 & \\
Always & & 68 \\
End point used $(n=166)$ & 41 & 74 \\
Complete stasis & 45 & 24 \\
Sluggish flow & 14 & \\
Pruned tree &
\end{tabular}

$n$ Number of respondents who answered the specific question

Table 5 Number of UFE treatments per category for centres with and without a dedicated UFE Web site

\begin{tabular}{lcc}
\hline No. of UFE procedures/year & \multicolumn{2}{c}{$\%$ UFE Web site presence $(n)$} \\
\cline { 2 - 3 } & No $(136)$ & Yes $(31)$ \\
\hline$\leq 10$ & $41(56)$ & $13(4)$ \\
10 to $<50$ & $51(69)$ & $61(19)$ \\
50 to $<100$ & $6(8)$ & $10(3)$ \\
100 to $<200$ & $1(2)$ & $10(3)$ \\
$\geq 200$ & $1(1)$ & $7(2)$ \\
\hline
\end{tabular}

advocated widely to properly map and follow-up a UFE candidate, only $56 \%$ of centres employed a pre-UFE MRI-planning protocol. Postprocedural MRI follow-up was even more disappointing, probably related to the poor follow-up intervals as stated previously. Contrastenhanced MRI is by all means the only reliable imaging modality to obviate sufficient devascularisation, e.g., a technically successful embolisation.

The fact that most centres $(81 \%)$ used a unifemoral arterial access for UFE minimizes the concern about potential adverse events occurring at the puncture sites, and using the Waltman loop manoeuvre might also be of great help in cases of steep aortic bifurcation issues. Although $66 \%$ of interventional radiologists do not use microcatheters, or use them only if necessary, coaxial use of microcatheters might be important to avoid vascular spasms, resulting in inadequate devascularisation of fibroids, thus leading to inferior clinical results. The chosen embolisation end point may depend on the type of embolisation material 
used. In the minority of centres, the pruned-tree appearance on fluoroscopic images is still used as the end point indicator. Sluggish flow in the uterine artery, e.g., the ShlanskyGoldberg method (stasis during five heartbeats) [17], was employed in equal frequency as total stasis of contrast medium in the uterine artery. Concerning embolisation material, no solid conclusive data are available to date on superior clinical outcome after UFE with a certain type embolic agent, although studies are pointing in the direction of calibrated microspheres [18-21]. It was therefore interesting to notice that a large majority of centres (77\%) favoured the use of these microspheres. Gelatin sponge was still employed in $1 \%$ of the centres. Although the role of the industry in promoting spherical embolic agents cannot be underestimated, the advantages of calibrated microspheres during UFE procedures, especially when using microcatheters, are evident. Less clogging of microcatheters and better prediction of the level of devascularisation might result in a smooth, swift, and successful UFE procedure.

Some treatment centres have dedicated fibroid clinics for preprocedural consultation and postembolisation clinical and radiologic follow-up. These centres of excellence often employ well-designed and properly executed public relations focused on potential patients and referring physicians. Kroencke stated the importance of using the media to enhance patient awareness of treatment options [22]. We should not underestimate the inventiveness of modern patients in their search for alternative treatment options independent from their treating physician. Not only the Internet, but also magazines, radio, and television, can be used by treatment centres to reach potential patients. The creation of an interactive Web site is a unique opportunity to do so. This study indicated that treatment centres operating a Web site containing dedicated UFE information performed more procedures than centres that do not use Web site possibilities, thus emphasizing the efficacy of such strategies. However, the presence of such a Web site might not be the only explanation for this finding.

Another important point in building a UFE practice is that interventional radiologists must become accustomed to treating patients in a clinical environment. UFE treatments must be performed by a multidisciplinary team, including gynaecologists and anaesthesiologists. It is extremely important, as quoted by Keeling et al., that interventional radiologists see the patient in the first place instead of a uterine fibroid that must be embolised [23]. Although quality of care is becoming increasingly important, it is no longer acceptable to meet the patient in the angiographic suite for the first time, perform the embolisation procedure, and never see her again.

Concerning new therapeutic developments, it was amazing to notice that Magnetic Resonance-guided Focused Ultrasound was categorized as a "very promising" respectively "promising" new treatment option by only $5 \%$ respectively $30 \%$ of the centres. Thirty-six percent did not see any role for MRgFUS in the future. However, recent papers show that MRgFUS can be a treatment option with satisfying results for a selection of patients [24-26]. The low levels of confidence we found might be partially biased by uneasy feelings toward a possible competitive treatment for UFE. Another reason could be that MRgFUS devices are expensive to acquire and therefore many of the respondents' facilities will never have the opportunity to obtain one. Moreover, current focused ultrasound technology can treat only relatively small volumes of fibroid tissue at a time, and respondents might see this is an essential factor limiting the number of patients that can be treated with this technique.

A limitation of this study was the low response rate (21.4\%). All CIRSE members received an invitation to participate in this online questionnaire; however, only a relatively small percentage responded. Therefore, we do not have the illusion of possessing a solid and complete data set on this subject because not all centres performing UFE completed the questionnaire. A low response rate is always a problem when conducting a survey, and it tends to be even lower when using an electronic survey instead of a survey sent by postal mail $[27,28]$. Therefore, the results published here are not an absolute view on UFE practice in Europe. It is possible that the nonresponding interventional centres do not perform UFE except, for instance, only nonvascular interventions. Another possibility is that some of the centres performing UFE are not members of CIRSE and therefore did not receive a survey invitation. Moreover, a key issue in survey research is nonresponse bias, which occurs when respondents differ in meaningful ways from nonrespondents. It is possible that the responders are more actively involved in this subject than nonresponders and are therefore more willing to participate in this survey.

We conclude with the statement that UFE as an alternative treatment for hysterectomy or myomectomy is widespread in Europe. However, the impact on the management of the patient with symptomatic fibroid seemed, according to the overall numbers of UFE procedures, disappointing. A more active attitude toward clinical IR, together with effective public relations, might establish a more solid fundament for UFE treatment in the future.

Acknowledgments We thank the CIRSE Central Office for their support.

Conflict of interest The authors declare that they have no conflict of interest.

Open Access This article is distributed under the terms of the Creative Commons Attribution Noncommercial License which permits any noncommercial use, distribution, and reproduction in any medium, provided the original author(s) and source are credited. 


\section{Appendix}

\begin{tabular}{|c|c|c|}
\hline \multicolumn{3}{|l|}{ Uterine Fibroid Embolisation Survev Europe } \\
\hline \multicolumn{3}{|l|}{$\underline{\text { Demographic information }}$} \\
\hline \multicolumn{3}{|l|}{$\begin{array}{l}\text { Name institution: } \\
\text { Name respondent: } \\
\text { In which country are you working: } \\
\text { In which city are you working: }\end{array}$} \\
\hline Do you perform Uterine Fibroid Embolisation (UFE)? & $\square$ & $\square$ No \\
\hline \multicolumn{3}{|l|}{ Interventional group } \\
\hline How many interventionalists are in your group? & $\begin{array}{l}\square \\
\square \\
\square \\
\square \\
\square\end{array}$ & $\begin{array}{l}\text { I am alone } \\
1 \text { physician } \\
2 \text { physicians } \\
3 \text { physicians } \\
4 \text { physicians }\end{array}$ \\
\hline \multicolumn{3}{|l|}{ How many interventionalists in your group perform UFE: } \\
\hline \multicolumn{3}{|l|}{ When did your group start performing UFE (year): } \\
\hline \multicolumn{3}{|l|}{ How many procedures does your group perform annually: } \\
\hline \multicolumn{3}{|l|}{$\underline{\text { Patient care }}$} \\
\hline $\begin{array}{l}\text { Could you indicate the percentages of referrals } \\
\text { (adding up to } 100 \% \text { ): }\end{array}$ & & $\begin{array}{l}\text { (patient): } \\
\text { aecology: } \\
\text { eral Practitioner: }\end{array}$ \\
\hline Who provides the pre-treatment care? & & $\begin{array}{l}\text { Self (radiologist) } \\
\text { Gynaecologist } \\
\text { Combination }\end{array}$ \\
\hline Who provides after care? & & $\begin{array}{l}\text { Self (radiologist) } \\
\text { Gynaecologist } \\
\text { Combination }\end{array}$ \\
\hline \multicolumn{3}{|l|}{ Procedure } \\
\hline Pain management: & $\begin{array}{l}\square \\
\square \\
\square\end{array}$ & $\begin{array}{l}\text { PCA (patient controlled } \\
\text { analgesia) } \\
\text { Epidural } \\
\text { Other: }\end{array}$ \\
\hline Which vascular access site do you prefer? & & $\begin{array}{l}\text { Unifemoral } \\
\text { Bifemoral } \\
\text { Other: }\end{array}$ \\
\hline Do you use microcatheters? & & $\begin{array}{l}\text { Never } \\
\text { Seldom } \\
\text { Regularly } \\
\text { Always }\end{array}$ \\
\hline Which embolic agent do you use? & $\frac{\square}{\square}$ & $\begin{array}{l}\text { Gelfoam } \\
\text { Spherical } \\
\text { Non-spherical }\end{array}$ \\
\hline What do you use as endpoint during your procedures? & $\begin{array}{l}\square \\
\square\end{array}$ & $\begin{array}{l}\text { Complete stasis } \\
\text { Sluggish flow } \\
\text { Pruned tree }\end{array}$ \\
\hline How long is the hospital stay? & $\begin{array}{l}\square \\
\square \\
\square \\
\square \\
\square\end{array}$ & $\begin{array}{l}\text { Outpatient basis } \\
1 \text { night stay } \\
2 \text { nights stay } \\
3 \text { nights stay } \\
4 \text { nights stay } \\
5 \text { nights stay }\end{array}$ \\
\hline What is your follow-up schedule? & $\begin{array}{l}\square \\
\square \\
\square \\
\square\end{array}$ & $\begin{array}{l}\text { No follow-up } \\
3 \text { months } \\
6 \text { months } \\
12 \text { months } \\
>12 \text { months }\end{array}$ \\
\hline When do you perform an MRI scan? & $\begin{array}{l}\square \\
\square \\
\square \\
\square \\
\square\end{array}$ & $\begin{array}{l}\text { Pre-procedural } \\
3 \text { months post-procedural } \\
6 \text { months post-procedural } \\
9 \text { months post-procedural } \\
12 \text { months post-procedural } \\
>12 \text { months post-procedural }\end{array}$ \\
\hline \multicolumn{3}{|l|}{$\underline{\text { General }}$} \\
\hline Does your institution have a website? & & $\begin{array}{l}\text { No } \\
\text { Yes: }\end{array}$ \\
\hline \multicolumn{3}{|l|}{ How many UFE procedures do you expect for 2009 : } \\
\hline What do you expect of MRI-guided focused ultrasound? & $\begin{array}{l}\square \\
\square \\
\square\end{array}$ & $\begin{array}{l}\text { Very promising } \\
\text { Promising } \\
\text { Nothing } \\
\text { Do not know }\end{array}$ \\
\hline
\end{tabular}

\section{References}

1. Stewart EA (2001) Uterine fibroids. Lancet 357(9252):293-298

2. Ravina JH, Herbreteau D, Ciraru-Vigneron N et al (1995) Arterial embolisation to treat uterine myomata. Lancet 346(8976): 671-672

3. Bradley LD (2009) Uterine fibroid embolization: a viable alternative to hysterectomy. Am J Obstet Gynecol 201:127-135

4. Ravina JH, Bouret JM, Fried D et al (1995) Value of preoperative embolization of uterine fibroma: report of a multicenter series of 31 cases. Contracept Fertil Sex 23:45-49

5. Gupta JK, Sinha A, Lumsden MA et al (2006) Uterine artery embolization for symptomatic uterine fibroids. Cochrane Database Syst Rev (1). Art. No.: CD 005073. doi:10.1002/ 14651858.CD005073.pub2.

6. Marshburn PB, Matthews ML, Hurst BS (2006) Uterine artery embolization as a treatment option for uterine myomas. Obstet Gynecol Clin North Am 33:125-144

7. Dutton S, Hirst A, McPherson K et al (2007) A UK multicentre retrospective cohort study comparing hysterectomy and uterine artery embolisation for the treatment of symptomatic uterine fibroids (HOPEFUL study): main results on medium-term safety and efficacy. BJOG 114:1340-1351

8. Hirst A, Dutton S, Wu O et al (2008) A multi-centre retrospective cohort study comparing the efficacy, safety and cost-effectiveness of hysterectomy and uterine artery embolisation for the treatment of symptomatic uterine fibroids. The HOPEFUL study. Health Technol Assess 12:1-248, iii

9. Edwards RD, Moss JG, Lumsden MA et al (2007) Uterine-artery embolization versus surgery for symptomatic uterine fibroids. N Engl J Med 356:360-370

10. Hehenkamp WJ, Volkers NA, Birnie E et al (2008) Symptomatic uterine fibroids: treatment with uterine artery embolization or hysterectomy-results from the randomized clinical Embolisation versus Hysterectomy (EMMY) Trial. Radiology 246:23-32

11. Pinto I, Chimeno P, Romo A et al (2003) Uterine fibroids: uterine artery embolization versus abdominal hysterectomy for treatment - a prospective, randomized, and controlled clinical trial. Radiology 226:425-431

12. Mara M, Maskova J, Fucikova $Z$ et al (2008) Midterm clinical and first reproductive results of a randomized controlled trial comparing uterine fibroid embolization and myomectomy. Cardiovasc Intervent Radiol 31:73-85

13. Lohle PN, Voogt MJ, De VJ et al (2008) Long-term outcome of uterine artery embolization for symptomatic uterine leiomyomas. J Vasc Interv Radiol 19:319-326

14. Popovic M, Berzaczy D, Puchner S et al (2009) Long-term quality of life assessment among patients undergoing uterine fibroid embolization. AJR Am J Roentgenol 193:267-271

15. Hovsepian DM, Siskin GP, Bonn J et al (2009) Quality improvement guidelines for uterine artery embolization for symptomatic leiomyomata. J Vasc Interv Radiol 20(Suppl):S193-S199

16. Bratby MJ, Belli AM (2008) Radiological treatment of symptomatic uterine fibroids. Best Pract Res Clin Obstet Gynaecol 22:717-734

17. Society of Interventional Radiology (2003) 28th Annual scientific meeting program. SIR, Fairfax, VA, pp 62-73

18. Spies JB, Allison S, Flick P et al (2004) Polyvinyl alcohol particles and tris-acryl gelatin microspheres for uterine artery embolization for leiomyomas: results of a randomized comparative study. J Vasc Interv Radiol 15:793-800

19. Siskin GP, Beck A, Schuster M et al (2008) Leiomyoma infarction after uterine artery embolization: a prospective randomized 
study comparing tris-acryl gelatin microspheres versus polyvinyl alcohol microspheres. J Vasc Interv Radiol 19:58-65

20. Rasuli P, Hammond I, Al-Mutairi B et al (2008) Spherical versus conventional polyvinyl alcohol particles for uterine artery embolization. J Vasc Interv Radiol 19:42-46

21. Golzarian J, Lang E, Hovsepian D et al (2006) Higher rate of partial devascularization and clinical failure after uterine artery embolization for fibroids with spherical polyvinyl alcohol. Cardiovasc Intervent Radiol 29:1-3

22. Kroencke TJ (2009) How to promote IR to patients and referring physicians. CIRSE annual meeting abstract book, Lisbon, Portugal, pp 237-278

23. Keeling AN, Reekers JA, Lee MJ (2009) The clinical practice of interventional radiology: a European perspective. Cardiovasc Intervent Radiol 32:406-411
24. Stewart EA, Gostout B, Rabinovici J et al (2007) Sustained relief of leiomyoma symptoms by using focused ultrasound surgery. Obstet Gynecol 110(2 Pt 1):279-287

25. LeBlang SD, Hoctor K, Steinberg FL (2010) Leiomyoma shrinkage after MRI-guided focused ultrasound treatment: report of 80 patients. AJR Am J Roentgenol 194:274-280

26. Fennessy FM, Tempany CM, McDannold NJ et al (2007) Uterine leiomyomas: MR imaging-guided focused ultrasound surgeryresults of different treatment protocols. Radiology 243:885-893

27. Akl EA, Maroun N, Klocke RA et al (2005) Electronic mail was not better than postal mail for surveying residents and faculty. J Clin Epidemiol 58:425-429

28. Seguin R, Godwin M, MacDonald S et al (2004) E-mail or snail mail? Randomized controlled trial on which works better for surveys. Can Fam Physician 50:414-419 